\title{
PSEUDOSPECTRA AND MATRIX BEHAVIOUR
}

\author{
THOMAS RANSFORD \\ Département de mathématiques, Université Laval \\ Québec (QC), Canada G1V 0A6 \\ E-mail: ransford@mat.ulaval.ca
}

\begin{abstract}
We study the extent to which the pseudospectra of a matrix determine other aspects of its behaviour, such as the growth of its powers and its unitary equivalence class.
\end{abstract}

1. Pseudospectra. The philosophy behind the interest in pseudospectra, and in particular the philosophy of Trefethen and Embree's book [15, is that, to predict accurately the growth of the norms of the powers of a matrix $A$, it is important to study not only the spectrum of $A$, but also its pseudospectra.

Let us illustrate this by an example. Consider the $32 \times 32$ tridiagonal matrix $A$ defined below.

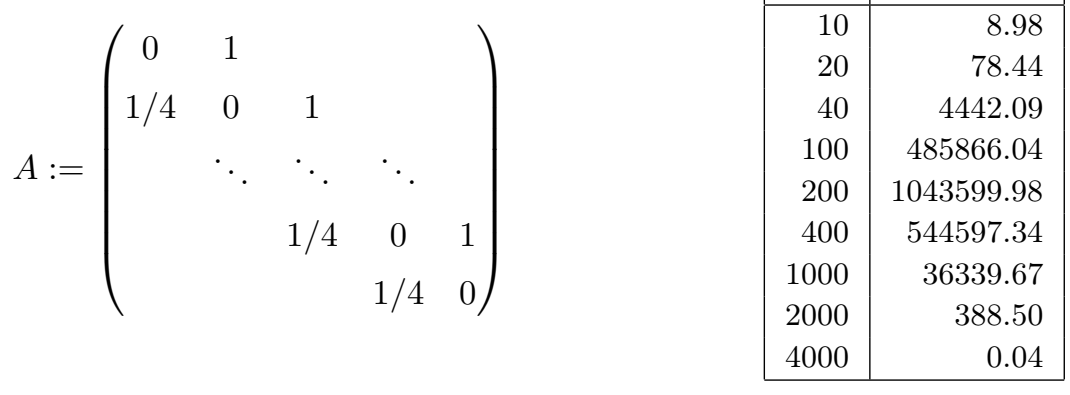

Its eigenvalues are $\cos (j \pi / 33), j=1, \ldots, 32$. In particular, the spectral radius of $A$ is strictly less than 1 , so we should expect that $A^{n} \rightarrow 0$ as $n \rightarrow \infty$. However, as shown in the table on the right, the operator norms $\left\|A^{n}\right\|$ (defined with respect to the usual Euclidean norm on vectors) actually become very large before finally decaying. This sort of transient behaviour is significant in applications, and it is important to understand it and predict when it will happen.

2010 Mathematics Subject Classification: Primary 15A18; Secondary 16R30.

Key words and phrases: matrix, pseudospectra, singular value, trace identity, unitary equivalence. The paper is in final form and no version of it will be published elsewhere. 
One way to do this is by looking at the resolvent $(A-z I)^{-1}$. Given an $N \times N$ matrix $A$ with eigenvalues in the unit disk, we always have

$$
M \leq \sup _{n}\left\|A^{n}\right\| \leq e N M
$$

where $M:=\sup _{|z|>1}\left\|(A-z I)^{-1}\right\|(|z|-1)$. The left-hand inequality in (1) is elementary: just expand $(A-z I)^{-1}$ as a Laurent series and use the triangle inequality. The right-hand inequality is a version of the Kreiss matrix theorem [9, 13]. At first sight, it is not obvious that $M$ is any easier to compute than $\left\|A^{n}\right\|$. However, using the fact that

$$
\left\|(A-z I)^{-1}\right\|=1 / s_{\min }(A-z I),
$$

where $s_{\min }$ denotes the minimum singular value, one can in fact compute resolvent norms very rapidly. The Matlab toolbox EigTool produces pictures of level curves of the norm of the resolvent. For instance, with $A$ as in the example above, we obtain the contour maps below (the dots are the eigenvalues of $A$, and the unit circle has been drawn in).
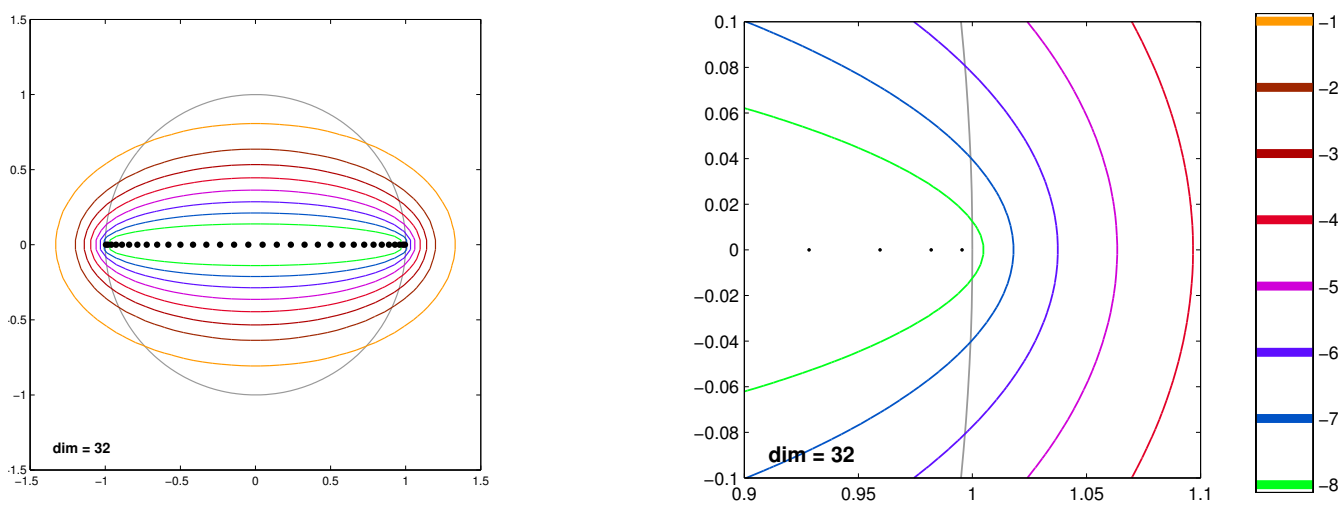

Fig. 1. Pseudospectra of $A:=\operatorname{triadiag}\left(32, \frac{1}{4}, 0,1\right)$

Looking at the right-hand picture at $z=1.01$, we can read off that $\left\|(A-z I)^{-1}\right\|>10^{7}$, and so, by the easy half of (1), it follows that $\sup _{n}\left\|A^{n}\right\|>10^{5}$, all this without costly computation of matrix powers.

The level curves of the resolvent (or rather, their interiors) are called pseudospectra. More precisely, for each $\epsilon>0$, the $\epsilon$-pseudospectrum of $A$ is defined to be

$$
\sigma_{\epsilon}(A):=\left\{z \in \mathbf{C}:\left\|(A-z I)^{-1}\right\|>1 / \epsilon\right\} .
$$

The book of Trefethen and Embree [15] gives an extensive account of the theory of pseudospectra, as well as applications in numerous fields, including atmospheric science, control theory, ecology, hydrodynamic stability, lasers, magnetohydrodynamics, Markov chains, matrix iterations, rounding error analysis, operator theory, quantum mechanics, and numerical solution of differential equations.

The general philosophy is that pseudospectra tell us more about the behaviour of (non-normal) matrices than spectra. This article, which draws substantially on the papers [5, 11], is intended as a survey of the author's recent researches into the precise nature of the link between the pseudospectra of a matrix and other aspects of its behaviour. 
2. Do pseudospectra determine matrix behaviour? Let $A$ and $B$ be two $N \times N$ matrices with identical pseudospectra. In other words, they satisfy the relation

$$
\left\|(A-z I)^{-1}\right\|=\left\|(B-z I)^{-1}\right\| \quad(z \in \mathbf{C}) .
$$

What other properties must they share? In particular:

(i) Does (3) imply that $\left\|A^{n}\right\|=\left\|B^{n}\right\|$ for all $n$ ?

(ii) Can we even go as far as to conclude that $A$ and $B$ are unitarily equivalent?

Notice that this is a bit different from the usual sort of problem in this subject, where typically the hypothesis is an upper bound for $\left\|(A-z I)^{-1}\right\|$, and the conclusion would be an upper bound for $\left\|A^{n}\right\|$. Here, we are assuming an exact knowledge of the resolvent norm, and the desired conclusion is correspondingly more ambitious.

Obviously questions (i) and (ii) have a positive answer if $N=1$, and it is a simple exercise to see that this remains true if $N=2$.

However, for $N \geq 3$ the answer to (ii) is a resounding "no". Here is a simple example. Let $A:=\operatorname{diag}(1,1,0)$ and $B:=\operatorname{diag}(1,0,0)$. A calculation shows that $\left\|(A-z I)^{-1}\right\|=$ $\max \left\{|z-1|^{-1},|z|^{-1}\right\}=\left\|(B-z I)^{-1}\right\|$, so $(3)$ is certainly satisfied. However, $A$ and $B$ are of different rank, so they are not even similar, let alone unitarily equivalent.

Now we turn to question (i). We begin with a positive result in this direction. The following theorem, which is well known, is a consequence of results relating pseudospectra to numerical range (see [15, §17]). For convenience, we give a direct proof.

Theorem 2.1. Let $A$ and $B$ be $N \times N$ matrices satisfying (3). Then

$$
1 / 2 \leq\|A\| /\|B\| \leq 2
$$

Proof. Let $\xi \in \mathbf{C}^{N}$ with $\|\xi\|_{2}=1$. Then, as $z \rightarrow 0$, we have

$$
\left\langle(I-z A)^{-1} \xi, \xi\right\rangle=1+z\langle A \xi, \xi\rangle+O\left(|z|^{2}\right) .
$$

On the other hand, we also have

$$
\left|\left\langle(I-z A)^{-1} \xi, \xi\right\rangle\right| \leq\left\|(I-z A)^{-1}\right\|=\left\|(I-z B)^{-1}\right\| \leq 1+|z|\|B\|+O\left(|z|^{2}\right) .
$$

Comparing the two, it follows that $|\langle A \xi, \xi\rangle| \leq\|B\|$. Hence also $\left|\left\langle A^{*} \xi, \xi\right\rangle\right| \leq\|B\|$. Hence also $\left|\left\langle H_{j} \xi, \xi\right\rangle\right| \leq\|B\|$, where $H_{1}:=\left(A+A^{*}\right) / 2$ and $H_{2}:=\left(A-A^{*}\right) / 2 i$. Now $H_{1}, H_{2}$ are self-adjoint matrices, so there exist $\xi_{1}, \xi_{2} \in \mathbf{C}^{N}$ of norm one such that $\mid\left\langle H_{j} \xi_{j}, \xi_{j}\right\rangle=\left\|H_{j}\right\|$. Thus $\left\|H_{j}\right\| \leq\|B\|$ for $j=1,2$, and finally $\|A\|=\left\|H_{1}+i H_{2}\right\| \leq\left\|H_{1}\right\|+\left\|H_{2}\right\| \leq 2\|B\|$, giving the right-hand side of (4). The left-hand side follows by symmetry.

It turns out that this result cannot be substantially improved. Greenbaum and Trefethen [7] gave an example of a pair of matrices $A, B$ satisfying (3) such that $\|A\| \neq\|B\|$, thereby answering question (i) in the negative. Here we present a generalization of their construction which further shows that the constant 2 appearing in Theorem 2.1 is sharp.

THEOREM 2.2. For each $N \geq 7$, there exist $N \times N$ matrices $A, B$ satisfying (3) such that

$$
\|A\|=1 \quad \text { and } \quad\|B\|=2 \cos \left(\frac{\pi}{N-1}\right) .
$$


Proof. Let $S_{n}$ denote the $n \times n$ shift matrix, with 1's just above the main diagonal and 0's elsewhere. Set $A:=S_{N-2} \oplus S_{2}$ and $B:=S_{N-2} \oplus \beta S_{2}$, where $\beta>1$ is a constant to be chosen later. Clearly $\|A\|=1$ and $\|B\|=\beta$. To verify (3), it suffices to show that

$$
\begin{aligned}
\left\|\left(I-z S_{2}\right)^{-1}\right\| & \leq\left\|\left(I-z S_{N-2}\right)^{-1}\right\| & & (z \in \mathbf{C}), \\
\left\|\left(I-z \beta S_{2}\right)^{-1}\right\| & \leq\left\|\left(I-z S_{N-2}\right)^{-1}\right\| & & (z \in \mathbf{C}) .
\end{aligned}
$$

Now, for $n=2$, a direct calculation gives

$$
\left\|\left(I-z S_{2}\right)^{-1}\right\|=\frac{|z|}{2}+\sqrt{1+\frac{|z|^{2}}{4}} \leq 1+\frac{|z|}{2}+\frac{|z|^{2}}{8} \quad(z \in \mathbf{C}) .
$$

For $n \geq 3$, we have the lower bound

$$
\left\|\left(I-z S_{n}\right)^{-1}\right\| \geq 1+|z| \cos \left(\frac{\pi}{n+1}\right)+|z|^{2} \cos \left(\frac{2 \pi}{n+1}\right) \quad(z \in \mathbf{C}) .
$$

To see this, consider the vector $\xi \in \mathbf{C}^{n}$ given by $\xi_{j}:=\sin (j \pi /(n+1)), j=1, \ldots, n$. It is an eigenvector of $S_{n}^{k}+S_{n}^{* k}$ for each $k \in\{1, \ldots, n-1\}$, with eigenvalue $2 \cos (k \pi /(n+1))$. Also $\xi_{j} \geq 0$ for all $j$. Hence, for $t \geq 0$,

$$
\begin{aligned}
\left\langle\left(I-t S_{n}\right)^{-1} \xi, \xi\right\rangle & =\sum_{k=0}^{n-1} t^{k}\left\langle S_{n}^{k} \xi, \xi\right\rangle \geq \sum_{k=0}^{2} t^{k}\left\langle S_{n}^{k} \xi, \xi\right\rangle \\
& =\left(1+t \cos \left(\frac{\pi}{n+1}\right)+t^{2} \cos \left(\frac{2 \pi}{n+1}\right)\right)\|\xi\|_{2}^{2} .
\end{aligned}
$$

This proves (5) when $z$ is positive, and the general case then follows, because $S_{n}$ is unitarily equivalent to $e^{i \theta} S_{n}$ for all $\theta$.

Returning to our construction, we now see that it suffices that

$$
1+\frac{\beta|z|}{2}+\frac{\beta^{2}|z|^{2}}{8} \leq 1+|z| \cos \left(\frac{\pi}{N-1}\right)+|z|^{2} \cos \left(\frac{2 \pi}{N-1}\right) \quad(z \in \mathbf{C}) .
$$

This will hold provided that $\beta \leq 2 \cos (\pi /(N-1))$ and $\beta^{2} \leq 8 \cos (2 \pi /(N-1))$. If we choose $\beta:=2 \cos (\pi /(N-1))$, then both constraints are satisfied provided that $N \geq 7$.

What about higher powers $\left\|A^{n}\right\| /\left\|B^{n}\right\|$ ? What is the analogue of (4) in this case? Actually, there isn't one. This is because of the following surprising result, proved in [11. Recall that a positive sequence $\left(\alpha_{n}\right)$ is submultiplicative if $\alpha_{n+m} \leq \alpha_{n} \alpha_{m}$ for all $n, m$.

THEOREM 2.3. Let $N \geq 7$ and let $\left(\alpha_{n}\right)$ and $\left(\beta_{n}\right)$ be arbitrary submultiplicative sequences. Then there exist $N \times N$ matrices $A, B$ satisfying (3) such that

$$
\left\|A^{n}\right\|=\alpha_{n} \quad \text { and } \quad\left\|B^{n}\right\|=\beta_{n} \quad(2 \leq n \leq(N-3) / 2) .
$$

Proof. This is similar in spirit to the previous proof, but this time using weighted shifts. For the details, we refer to [11, Theorem 1.1].

3. Super-identical pseudospectra. The results in the previous section indicate that pseudospectra do not determine matrix behaviour, at least when 'behaviour' is interpreted in the sense of the questions (i) and (ii). An examination of the examples reveals that this is because some parts of a matrix can 'hide behind' others in such a way that they cannot be detected using resolvent norms alone. A more powerful tool is needed. 
One possibility, suggested by results such as the Hille-Yosida theorem, is to consider the generalized resolvent norms $\left\|(A-z I)^{-k}\right\|$ for $k \geq 1$. However, this is unsatisfactory for at least two reasons. Firstly, it is still insufficient to distinguish between the simple matrix pair $A:=\operatorname{diag}(1,1,0)$ and $B:=\operatorname{diag}(1,0,0)$. Secondly, and perhaps even more importantly, the whole point of pseudospectra is to avoid the costly computation of matrix powers, so it is illogical to incorporate powers as part of the definition.

In fact, these same practical considerations suggest another possibility. Recall that the singular values $s_{k}(A)$ of an $N \times N$ matrix $A$ are the square roots of the eigenvalues of $A A^{*}$, ordered so that $s_{1}(A) \geq s_{2}(A) \geq \cdots \geq s_{N}(A)$. In particular $s_{1}(A)=\|A\|$ and $s_{N}(A)=1 /\left\|A^{-1}\right\|$. The latter equation leads immediately to the formula (2), which is how resolvent norms are actually calculated in practice, the point being that singular values are relatively inexpensive to compute. The condition (3) that matrices $A$ and $B$ have identical pseudospectra can be expressed in the equivalent form:

$$
s_{N}(A-z I)=s_{N}(B-z I) \quad(z \in \mathbf{C}) .
$$

Viewed in this way, the condition has a natural extension. The following definition was formulated in [5], which is also the source for the results in the rest of this section.

Definition 3.1. Two $N \times N$ matrices $A$ and $B$ have super-identical pseudospectra if

$$
s_{k}(A-z I)=s_{k}(B-z I) \quad(z \in \mathbf{C}, k=1, \ldots, N) .
$$

Once again, we pose the following questions:

(i) Does (6) imply that $\left\|A^{n}\right\|=\left\|B^{n}\right\|$ for all $n$ ?

(ii) Can we even go as far as to conclude that $A$ and $B$ are unitarily equivalent?

To investigate these questions, it is convenient first to reformulate condition (6) in terms of trace identities. We write $\operatorname{tr}(A)$ to denote the trace of $A$.

TheOREm 3.2. Let $A$ and $B$ be $N \times N$ matrices. The following are equivalent:

- $A$ and $B$ have super-identical pseudospectra.

- $\operatorname{tr}\left(\left[(A-z I)\left(A^{*}-\bar{z} I\right)\right]^{k}\right)=\operatorname{tr}\left(\left[(B-z I)\left(B^{*}-\bar{z} I\right)\right]^{k}\right)$ for $z \in \mathbf{C}$ and $k \geq 0$.

- $\operatorname{tr}\left(\left[(A-z I)\left(A^{*}-\bar{z} I\right)\right]^{k}\right)=\operatorname{tr}\left(\left[(B-z I)\left(B^{*}-\bar{z} I\right)\right]^{k}\right)$ for $z \in \mathbf{C}$ and $k \in\{1, \ldots, N\}$.

Proof. By definition of singular value, condition (6) is equivalent to demanding that, for each $z \in \mathbf{C}$, the matrices $(A-z I)(A-z I)^{*}$ and $(B-z I)(B-z I)^{*}$ have the same eigenvalues (counting multiplicities). Now, if two $N \times N$ matrices $C$ and $D$ have the same eigenvalues, then clearly $\operatorname{tr}\left(C^{k}\right)=\operatorname{tr}\left(D^{k}\right)$ for all $k \geq 0$. Conversely, if $\operatorname{tr}\left(C^{k}\right)=\operatorname{tr}\left(D^{k}\right)$ for $k \in\{1, \ldots, N\}$, then, thanks to Newton's identities, $C$ and $D$ have the same eigenvalues.

The final condition in Theorem 3.2 is a set of identities between polynomials in $(z, \bar{z})$ of bidegree $(N, N)$. We therefore immediately obtain the following corollary.

Corollary 3.3. Let $F$ be a uniqueness set for polynomials in $(z, \bar{z})$ of bidegree $(N, N)$. Two $N \times N$ matrices $A$ and $B$ have super-identical pseudospectra iff

$$
s_{k}(A-z I)=s_{k}(B-z I) \quad(z \in F, k=1, \ldots, N) .
$$


The interest of this result is that the uniqueness sets $F$ may be finite. In fact it is easy to construct such sets having $(N+1)^{2}$ elements. Thus, despite initial appearances, the condition that $A, B$ have super-identical pseudospectra is actually a finite condition.

The next task is to find a more explicit expression for the polynomials in $(z, \bar{z})$ appearing in Theorem 3.2 . This is a combinatorial problem. It boils down to identifying the coefficients $f_{i j k}(X, Y)$ in the expression:

$$
\operatorname{tr}\left([(I+\xi X)(I+\eta Y)]^{k}\right)=\sum_{i, j} f_{i j k}(X, Y) \xi^{i} \eta^{j} .
$$

Let $W$ be the set of all words in two non-commuting variables $x, y$. Given $w \in W$ and $v \in W \backslash\{1\}$, we denote by $d_{v}(w)$ the number of times the word $v$ appears in $w$. Define

$$
g_{i j l}(X, Y):=\sum\left\{\operatorname{tr}(w(X, Y)): w \in W, d_{x}(w)=i, d_{y}(w)=j, d_{x y}(w)=l\right\} .
$$

TheOREm 3.4. For all $i, j, k \geq 0$, we have

$$
f_{i j k}(X, Y)=\sum_{l \geq 0}\left(\begin{array}{l}
k+l \\
i+j
\end{array}\right) g_{i j l}(X, Y)
$$

Also, for all $i, j, l \geq 0$, we have

$$
g_{i j l}(X, Y)=\sum_{k \geq 0}(-1)^{i+j+k+l}\left(\begin{array}{l}
i+j+1 \\
k+l+1
\end{array}\right) f_{i j k}(X, Y) .
$$

Proof. The first identity is proved using a non-commutative binomial theorem. The second one is deduced using an inversion formula. For the details, see [5, Theorem 2.7].

From this, we immediately deduce the following corollary.

Corollary 3.5. Let $A$ and $B$ be $N \times N$ matrices. The following are equivalent:

- $A$ and $B$ have super-identical pseudospectra.

- $g_{i j l}\left(A, A^{*}\right)=g_{i j l}\left(B, B^{*}\right)$ for all $i, j, l \geq 0$.

- $g_{i j l}\left(A, A^{*}\right)=g_{i j l}\left(B, B^{*}\right)$ for $i, j \in\{l, \ldots, N\}$ and $l \in\{1, \ldots, N\}$.

We can now give a partial affirmative answer to question (i), not just for powers, but for arbitrary polynomials. The following theorem should be contrasted with Theorem 2.3 .

Theorem 3.6. Let $A$ and $B$ be $N \times N$ matrices with super-identical pseudospectra. Then, for every polynomial $p(z)$,

$$
1 / \sqrt{N} \leq\|p(A)\| /\|p(B)\| \leq \sqrt{N}
$$

Proof. By Corollary 3.5. we have $g_{i j 0}\left(A, A^{*}\right)=g_{i j 0}\left(B, B^{*}\right)$ for all $i, j$, in other words $\operatorname{tr}\left(A^{* j} A^{i}\right)=\operatorname{tr}\left(B^{* j} B^{i}\right)$. It follows that, for any polynomial $p$, we have $\operatorname{tr}\left(p(A)^{*} p(A)\right)=$ $\operatorname{tr}\left(p(B)^{*} p(B)\right)$, or in other words, $\sum_{1}^{N} s_{k}^{2}(p(A))=\sum_{1}^{N} s_{k}^{2}(p(B))$. Hence

$$
\|p(A)\|^{2}=s_{1}^{2}(p(A)) \leq \sum_{k=1}^{N} s_{k}^{2}(p(A))=\sum_{k=1}^{N} s_{k}^{2}(p(B)) \leq N s_{1}^{2}(p(B))=N\|p(B)\|^{2} .
$$

Likewise $\|p(B)\|^{2} \leq N\|p(A)\|^{2}$. 
4. Examples in low dimensions. Can we replace $\sqrt{N}$ by 1 in the preceding theorem? In order to investigate this further, we explore in more detail what happens in low dimensions, namely $N=3,4$ (recall that the cases $N=1,2$ were already settled in \$2). To this end, we make use of an old result due to Frobenius [6, §3], which is very useful when working with trace identities. Though elementary, it does not appear to be so well known, so we digress a moment to discuss it.

Let $X_{1}, X_{2}, \ldots, X_{m}$ be $N \times N$ matrices. Let $\sigma \in S_{m}$ be a permutation, say $\sigma=$ $\left(a_{1} \ldots a_{i}\right)\left(b_{1} \ldots b_{j}\right) \ldots\left(c_{1} \ldots c_{k}\right)$ when written as a product of disjoint cycles. We define

$$
\operatorname{tr}_{\sigma}\left(X_{1}, \ldots, X_{m}\right):=\operatorname{tr}\left(X_{a_{1}} \cdots X_{a_{i}}\right) \operatorname{tr}\left(X_{b_{1}} \cdots X_{b_{j}}\right) \ldots \operatorname{tr}\left(X_{c_{1}} \cdots X_{c_{k}}\right) .
$$

The properties of trace ensure this is well defined. We write $\epsilon(\sigma)$ for the signature of $\sigma$.

Theorem 4.1 (Frobenius' identity). Let $X_{1}, \ldots, X_{N+1}$ be $N \times N$ matrices. Then

$$
\sum_{\sigma \in S_{N+1}} \epsilon(\sigma) \operatorname{tr}_{\sigma}\left(X_{1}, \ldots, X_{N+1}\right)=0
$$

If we take $X_{1}=\cdots=X_{N}=A$ and $X_{N+1}=B$, then Frobenius' identity becomes $\operatorname{tr}\left(p_{A}(A) B\right)=0$, where $p_{A}(z)$ is a polynomial of degree $N$ whose coefficients depend on $A$. As this holds for all $B$, it follows that $p_{A}(A)=0$. If $A$ has distinct eigenvalues, then necessarily these are roots of $p_{A}(z)$. Hence $p_{A}$ is a multiple of the characteristic polynomial of $A$, and the identity $p_{A}(A)=0$ is nothing other than the Cayley-Hamilton theorem. Thus Frobenius' identity can be regarded as a polarized form of the Cayley-Hamilton theorem, and indeed it can be proved this way (see [3]). Here we give a more direct proof.

Proof of Theorem 4.1. Since the expression (9) is multilinear in $X_{1}, \ldots X_{N+1}$, it suffices to prove it in the case when each $X_{i}$ is one of the basic matrices $E_{j k}$. Here $E_{j k}$ denotes the matrix which has a 1 in the $j k$-th entry and zeros everywhere else.

An elementary computation shows that

$$
\operatorname{tr}\left(E_{j_{1} k_{1}} E_{j_{2} k_{2}} \cdots E_{j_{l} k_{l}}\right)=\delta\left(k_{1}, j_{2}\right) \delta\left(k_{2}, j_{3}\right) \ldots \delta\left(k_{l}, j_{1}\right),
$$

where, as usual, $\delta(k, j):=1$ if $k=j$ and $:=0$ if $k \neq j$. Hence,

$$
\operatorname{tr}_{\sigma}\left(E_{j_{1} k_{1}}, \ldots, E_{j_{N+1} k_{N+1}}\right)=\delta\left(j_{\sigma(1)}, k_{1}\right) \delta\left(j_{\sigma(2)}, k_{2}\right) \ldots \delta\left(j_{\sigma(N+1)}, k_{N+1}\right) .
$$

Thus

$$
\sum_{\sigma \in S_{N+1}} \epsilon(\sigma) \operatorname{tr}_{\sigma}\left(E_{j_{1} k_{1}}, \ldots, E_{j_{N+1} k_{N+1}}\right)=\sum\left\{\epsilon(\sigma): j_{\sigma(i)}=k_{i} \text { for all } i\right\} .
$$

Now $k_{1}, \ldots, k_{N+1} \in\{1, \ldots, N\}$, so, by the pigeonhole principle, there exist distinct $p, q$ such that $k_{p}=k_{q}$. Let $\tau$ be the transposition $(p, q)$. Then $j_{\sigma(i)}=k_{i}$ for all $i$ iff $j_{\sigma \tau(i)}=k_{i}$ for all $i$. Also $\epsilon(\sigma)=-\epsilon(\sigma \tau)$. It follows that the last sum above is equal to minus itself, and is therefore zero. This completes the proof.

There is a sense in which every other trace identity for matrices is a consequence of Frobenius' identity. For more details about this converse result, which is due independently to Procesi and Razmyslov, see [4, 8, 10]. We do not need it here.

Returning now to pseudospectra, the following result improves Corollary 3.5 when $N=3$. 
TheOREM 4.2. The $3 \times 3$ matrices $A$ and $B$ have super-identical pseudospectra iff

$$
\begin{cases}\operatorname{tr} A^{i}=\operatorname{tr} B^{i}, & i=1,2,3, \\ \operatorname{tr}\left(A^{i} A^{* j}\right)=\operatorname{tr}\left(B^{i} B^{* j}\right), & 1 \leq i \leq j \leq 2 .\end{cases}
$$

Proof. By Corollary 3.5. the conditions are certainly necessary. To prove sufficiency, we need to show that $(A-z I)(A-z I)^{*}$ and $(B-z I)(B-z I)^{*}$ have the same eigenvalues for all $z \in \mathbf{C}$, and for this it suffices to show that

$$
\begin{aligned}
\operatorname{tr}\left((A-z I)\left(A^{*}-\bar{z} I\right)\right) & =\operatorname{tr}\left((B-z I)\left(B^{*}-\bar{z} I\right)\right), \\
\operatorname{tr}\left(\left[(A-z I)\left(A^{*}-\bar{z} I\right)\right]^{2}\right) & =\operatorname{tr}\left(\left[(B-z I)\left(B^{*}-\bar{z} I\right)\right]^{2}\right), \\
\operatorname{det}\left((A-z I)\left(A^{*}-\bar{z} I\right)\right) & =\operatorname{det}\left((B-z I)\left(B^{*}-\bar{z} I\right)\right) .
\end{aligned}
$$

The first and the third of these equations follow easily from $10 p$. The second would likewise follow if we knew that $\operatorname{tr}\left(A A^{*} A A^{*}\right)=\operatorname{tr}\left(B B^{*} B B^{*}\right)$. It is here that Frobenius' identity comes into play. Taking $X_{1}=X_{2}=A$ and $X_{3}=X_{4}=A^{*}$ in Theorem 4.1, we obtain

$$
\begin{aligned}
4 \operatorname{tr}\left(A A^{*} A A^{*}\right) & +2 \operatorname{tr}\left(A^{2} A^{* 2}\right)+4 \operatorname{tr}(A) \operatorname{tr}\left(A A^{* 2}\right)+4 \operatorname{tr}\left(A^{*}\right) \operatorname{tr}\left(A^{2} A^{*}\right) \\
& +\operatorname{tr}\left(A^{2}\right) \operatorname{tr}\left(A^{* 2}\right)+2 \operatorname{tr}^{2}\left(A A^{*}\right)+\operatorname{tr}^{2}(A) \operatorname{tr}\left(A^{* 2}\right) \\
& +\operatorname{tr}\left(A^{2}\right) \operatorname{tr}^{2}\left(A^{*}\right)+\operatorname{tr}^{2}(A) \operatorname{tr}^{2}\left(A^{*}\right)+4 \operatorname{tr}(A) \operatorname{tr}\left(A^{*}\right) \operatorname{tr}\left(A A^{*}\right)=0 .
\end{aligned}
$$

All the terms but the first are governed by 100 . Hence the first term is also.

Corollary 4.3. Let $A$ and $B$ be $3 \times 3$ matrices with super-identical pseudospectra. Then, for every polynomial $p(z)$, the matrices $p(A)$ and $p(B)$ have super-identical pseudospectra. In particular $\|p(A)\|=\|p(B)\|$.

Proof. The conditions 10 for $A, B$ imply the same conditions for $p(A), p(B)$.

Thus our question (i) has an affirmative answer if $N=3$. Next, we turn to $N=4$.

TheOREM 4.4. The $4 \times 4$ matrices $A$ and $B$ have super-identical pseudospectra iff

$$
\begin{cases}\operatorname{tr} A^{i}=\operatorname{tr} B^{i}, & i=1,2,3,4, \\ \operatorname{tr}\left(A^{i} A^{* j}\right)=\operatorname{tr}\left(B^{i} B^{* j}\right), & 1 \leq i \leq j \leq 3, \\ \operatorname{tr}\left(A A^{*} A A^{*}\right)=\operatorname{tr}\left(B B^{*} B B^{*}\right) . & \end{cases}
$$

Proof. This is similar to the proof of Theorem 4.2, though evidently more complicated. This time we need to use Frobenius' identity three times, with $\left(X_{1}, X_{2}, X_{3}, X_{4}, X_{5}\right)=$ $\left(A, A, A, A^{*}, A^{*}\right),\left(A, A, A^{*}, A^{*}, A A^{*}\right)$ and $\left(A, A, A, A^{*}, A^{* 2}\right)$. The details are omitted.

If we try to repeat the proof of Corollary 4.3 for $N=4$, we run up against an obstacle. It is not clear whether (11) implies $\operatorname{tr}\left(p(A) p(A)^{*} p(A) p(A)^{*}\right)=\operatorname{tr}\left(p(B) p(B)^{*} p(B) p(B)^{*}\right)$, even for $p(z)=z^{2}$. In fact, after playing with this for a while, we are actually led to the following counterexample, which shows finally that the answer to question (i) is negative. (This counterexample was already presented in [5], but with a different proof, and without any explanation of how it was derived.) 
TheOREM 4.5. Let $\alpha, \beta \in(\pi / 4]$ and let

$$
A:=\left(\begin{array}{cccc}
0 & \sec \alpha & 0 & 1 \\
0 & 0 & \sec \beta \csc \beta & 0 \\
0 & 0 & 0 & \csc \alpha \\
0 & 0 & 0 & 0
\end{array}\right) \quad \text { and } B:=\left(\begin{array}{cccc}
0 & \sec \beta & 0 & 1 \\
0 & 0 & \sec \alpha \csc \alpha & 0 \\
0 & 0 & 0 & \csc \beta \\
0 & 0 & 0 & 0
\end{array}\right) .
$$

Then $A, B$ have super-identical pseudospectra, and $\left\|A^{2}\right\| /\left\|B^{2}\right\|=\cos \alpha / \cos \beta$.

Proof. One checks that $A$ and $B$ satisfy (11), so they have super-identical pseudospectra. Also, a simple computation gives $\left\|A^{2}\right\|=\csc \alpha \sec \beta \csc \beta$ and $\left\|B^{2}\right\|=\csc \beta \sec \alpha \csc \alpha$, which proves the final statement in the theorem.

5. Unitary equivalence. In providing a negative answer to question (i), we have at the same time answered negatively question (ii): there exist $4 \times 4$ matrices with superidentical pseudospectra that are not unitarily equivalent. Actually, for question (ii) there is a simpler counterexample, which works even when $N=3$. Let

$$
A:=\left(\begin{array}{lll}
0 & 1 & 0 \\
0 & 0 & 2 \\
0 & 0 & 0
\end{array}\right),
$$

and let $B$ be the transpose of $A$. Then $A$ and $B$ have super-identical pseudospectra, because this is always true for mutually transpose matrices. But they are not unitarily equivalent, because $\operatorname{tr}\left(A A^{*} A^{2} A^{* 2}\right) \neq \operatorname{tr}\left(B B^{*} B^{2} B^{* 2}\right)$ (the first is 4 and the second is 16).

However, this is not the end of the story. Unitary equivalence can itself be characterized in terms of trace identities. The following theorem is due to Specht [12. Recall that $W$ denotes the set of all words in two non-commuting variables.

TheOREM 5.1. Two $N \times N$ matrices $A$ and $B$ are unitarily equivalent if and only if $\operatorname{tr} w\left(A, A^{*}\right)=\operatorname{tr} w\left(B, B^{*}\right)$ for every word $w \in W$.

This result bears a marked resemblance to Corollary 3.5. which provides an analogous characterization of matrices with super-identical pseudospectra. To further compare these two characterizations, it is convenient to introduce some notation. The following definition is taken from [5], which is also the source for the rest of this section.

Definition 5.2. Let $N \geq 1$ and let $X, Y$ be generic $N \times N$ matrices.

- $\mathcal{I}_{N}$ denotes the algebra generated by the polynomials $\{\operatorname{tr} w(X, Y): w \in W\}$.

- $\mathcal{F}_{N}$ denotes the subalgebra of $\mathcal{I}_{N}$ generated by the family $\left\{f_{i j k}(X, Y): i, j, k \geq 0\right\}$ defined in (7). Equivalently, it is the subalgebra generated by $\left\{g_{i j l}(X, Y): i, j, l \geq 0\right\}$ defined in (8).

The characterizations described above can be succinctly summarized as follows:

$A, B$ have super-identical pseudospectra $\Leftrightarrow f\left(A, A^{*}\right)=f\left(B, B^{*}\right)$ for all $f \in \mathcal{F}_{N}$,

$A, B$ are unitarily equivalent $\Leftrightarrow f\left(A, A^{*}\right)=f\left(B, B^{*}\right)$ for all $f \in \mathcal{I}_{N}$.

Also, the example given at the beginning of this section shows that $\operatorname{tr}\left(X Y X^{2} Y^{2}\right) \in \mathcal{I}_{3} \backslash \mathcal{F}_{3}$. In fact, it is known that $\mathcal{I}_{3}$ is generated by $\mathcal{F}_{3}$ and $\operatorname{tr}\left(X Y X^{2} Y^{2}\right)$ (see [14, Theorem 4.1]). For $N \geq 4$, the situation is much more complicated. It is known that a minimal generating 
set for $\mathcal{I}_{4}$ contains 32 elements, and for $\mathcal{I}_{5}$ this number rises to 173 (see [1]). From their definition, the algebras $\mathcal{F}_{4}$ and $\mathcal{F}_{5}$ have somewhat smaller generating sets. Thus, it would appear that the gap between $\mathcal{I}_{N}$ and $\mathcal{F}_{N}$ widens as $N$ grows. Theorem 4.5 lends support to this impression. The following result is therefore perhaps a little surprising.

TheOREm 5.3. For each $N$, the algebra $\mathcal{I}_{N}$ is algebraic over $\mathcal{F}_{N}$. In other words, given $h \in \mathcal{I}_{N}$, there exist $f_{0}, \ldots, f_{k} \in \mathcal{F}_{N}$, with $f_{0} \neq 0$, such that $f_{0} h^{k}+f_{1} h^{k-1}+\cdots+f_{k}=0$.

Proof. It is known that $\mathcal{I}_{N}$ has transcendence degree $N^{2}+1$ over $\mathbf{C}$ (see [2, pp.65,186]). The theorem amounts to proving that the same is true of $\mathcal{F}_{N}$. The most direct way to achieve this would be to exhibit an explicit set of $N^{2}+1$ algebraically independent elements of $\mathcal{F}_{N}$. We do not know how to do this. Instead, the proof involves a somewhat indirect estimation of transcendence degree using partial derivatives. For the details, we refer to [5, §4].

This algebraicity theorem has the following consequence for pseudospectra. We write $\mathbf{C}^{N \times N}$ for the set of $N \times N$ matrices with the usual topology and Lebesgue measure.

TheOREM 5.4. Given $N \geq 1$, there exist an integer $m$ and a closed subset $E$ of $\mathbf{C}^{N \times N}$ of measure zero such that every super-identical-pseudospectral equivalence class in $\mathbf{C}^{N \times N}$ is either contained in $E$ or is a union of at most $m$ unitary equivalence classes.

Proof. It is known that $\mathcal{I}_{N}$ is finitely generated (see [10, Theorem 3.4(a)]). Let $h_{1}, \ldots, h_{r}$ be a set of generators for $\mathcal{I}_{N}$. By Theorem 5.3. each $h_{i}$ is algebraic over $\mathcal{F}_{N}$, so satisfies a polynomial equation

$$
f_{i 0} h_{i}^{m_{i}}+f_{i 1} h_{i}^{m_{i}-1}+\cdots+f_{i m_{i}}=0,
$$

where $f_{i 0}, f_{i 1}, \ldots, f_{i m_{i}} \in \mathcal{F}_{N}$ and $f_{i 0} \neq 0$. Set $m:=m_{1} \ldots m_{r}$ and

$$
E:=\left\{A \in \mathbf{C}^{N \times N}: f_{10}\left(A, A^{*}\right) \cdots f_{r 0}\left(A, A^{*}\right)=0\right\} .
$$

Then $E$ is a closed set of measure zero (in fact it is a real-algebraic subvariety), and also it is a union of super-identical-pseudospectral equivalence classes.

It remains to show that, if $A_{1}, \ldots, A_{m+1} \in \mathbf{C}^{N \times N} \backslash E$ have super-identical pseudospectra, then at least two of them are unitarily equivalent. For each $i, j$, we have

$$
f_{i 0}\left(A_{j}, A_{j}^{*}\right) h_{i}\left(A_{j}, A_{j}^{*}\right)^{m_{i}}+f_{i 1}\left(A_{j}, A_{j}^{*}\right) h_{i}\left(A_{j}, A_{j}^{*}\right)^{m_{i}-1}+\cdots+f_{i m_{i}}\left(A_{j}, A_{j}^{*}\right)=0 .
$$

Since $f_{i k} \in \mathcal{F}_{N}$, the coefficients $f_{i k}\left(A_{j}, A_{j}^{*}\right)$ are independent of $j$. Also $f_{i 0}\left(A_{j}, A_{j}^{*}\right) \neq 0$ for all $j$. Thus the numbers $h_{i}\left(A_{1}, A_{1}^{*}\right), \ldots, h_{i}\left(A_{m+1}, A_{m+1}^{*}\right)$ all satisfy the same polynomial of degree $m_{i}$. Denote by $R_{i}$ the set of roots of this polynomial. Thus

$$
\left(h_{1}\left(A_{j}, A_{j}^{*}\right), \ldots, h_{r}\left(A_{j}, A_{j}^{*}\right)\right) \in R_{1} \times \cdots \times R_{r} \quad(j=1, \ldots, m+1) .
$$

The cardinality of $R_{1} \times \cdots \times R_{r}$ is at most $m_{1} \cdots m_{r}=m$, so by the pigeonhole principle there exist distinct $j, k \in\{1, \ldots, m+1\}$ such that

$$
\left(h_{1}\left(A_{j}, A_{j}^{*}\right), \ldots, h_{r}\left(A_{j}, A_{j}^{*}\right)\right)=\left(h_{1}\left(A_{k}, A_{k}^{*}\right), \ldots, h_{r}\left(A_{k}, A_{k}^{*}\right)\right) .
$$

Because the $h_{i}$ generate $\mathcal{I}_{N}$, we deduce that $h\left(A_{j}, A_{j}^{*}\right)=h\left(A_{k}, A_{k}^{*}\right)$ for all $h \in \mathcal{I}_{N}$. Therefore $A_{j}$ and $A_{k}$ are unitarily equivalent. 
Theorem 5.4 can be viewed as a sort of discreteness theorem. For example, given $A \in \mathbf{C}^{N \times N} \backslash E$, if $B$ is any matrix satisfying $(6)$ and $p(z)$ is any polynomial, then not only must we have $1 / \sqrt{N} \leq\|p(A)\| /\|p(B)\| \leq \sqrt{N}$, but there are at most $m$ possibilities for the value of $\|p(B)\|$.

It seems likely that Theorem 5.4 is actually true with $E=\emptyset$. The most natural way to prove this would seem to be via an improved version of Theorem 5.3, in which 'algebraic' is replaced by 'integral' (this means that $f_{0}$ may be taken to be 1 ). Unfortunately, this 'improved version' is known to be false. It is shown in [5, Theorem 4.12] that $\operatorname{tr}\left(X^{2} Y^{2} X^{2} Y^{2}\right)$ is not integral over $\mathcal{F}_{4}$. The problem of whether we can take $E=\emptyset$ is still open.

6. Conclusion. Modeling of experiments by linear dynamical systems gives rise to the problem of estimation of $\left\|A^{n}\right\|$ or of $\left\|e^{t A}\right\|$. For non-normal matrices, standard eigenvalue analysis tells only part of the story, and can sometimes even be misleading. We get more information by looking at level curves of the resolvent (pseudospectra), which can be rapidly computed using singular values. However, pseudospectra alone are not sufficient to determine matrix behaviour, and the notion of super-identical pseudospectra leads to more satisfactory results. The challenge now is to convert these results into practical algorithms.

Acknowledgments. This paper is based on a lecture delivered at the $19^{\text {th }}$ International Conference on Banach Algebras, held at Będlewo, July 14-24, 2009. The support for the meeting by the Polish Academy of Sciences, the European Science Foundation under the ESF-EMS-ERCOM partnership, and the Faculty of Mathematics and Computer Science of the Adam Mickiewicz University at Poznań is gratefully acknowledged. This research was also supported by grants from NSERC (Canada), FQRNT (Québec) and the Canada research chairs program.

\section{References}

[1] D. Ž. Doković, Poincaré series of some pure and mixed trace algebras of two generic matrices, J. Algebra 309 (2007), 654-671.

[2] V. Drensky and E. Formanek, Polynomial Identity Rings, Birkhaüser, Basel, 2004.

[3] E. Formanek, Polynomial identities and the Cayley-Hamilton theorem, Math. Intelligencer 11 (1989), 37-39.

[4] E. Formanek, The Polynomial Identities and Invariants of $n \times n$ Matrices, American Mathematical Society, Providence, RI, 1991.

[5] M. Fortier Bourque and T. Ransford, Super-identical pseudospectra, J. London Math. Soc. 79 (2009), 511-528.

[6] F. G. Frobenius, Über die Primfactoren der Gruppendeterminante, Sitz. Kön. Preuss. Akad. Wiss. Berlin (1896), 1343-1382.

[7] A. Greenbaum and L. N. Trefethen, Do the pseudospectra of a matrix determine its behavior?, Technical Report TR 93-1371, Computer Science Department, Cornell University, 1993. 
[8] U. Leron, Trace identities and polynomial identities of $n \times n$ matrices, J. Algebra 42 (1976), 369-377.

[9] R. LeVeque and L. N. Trefethen, On the resolvent condition in the Kreiss matrix theorem, BIT 24 (1984), 584-591.

[10] C. Procesi, The invariant theory of $n \times n$ matrices, Adv. Math. 19 (1976), 306-381.

[11] T. Ransford, Pseudospectra and power growth, SIAM J. Matrix Anal. Appl. 29 (2007), 699-711.

[12] W. Specht, Zur Theorie der Matrizen II, Jber. Deutsch. Math. Verein. 50 (1940), 19-23.

[13] M. N. Spijker, On a conjecture by LeVeque and Trefethen related to the Kreiss matrix theorem, BIT 31 (1991), 551-555.

[14] Y. Teranishi, The ring of invariants of matrices, Nagoya Math. J. 104 (1986), 149-161.

[15] L. N. Trefethen and M. Embree, Spectra and Pseudospectra, Princeton University Press, Princeton, 2005. 